\title{
Molecular Dynamics Study of Homogeneous Nucleation and Growth in a Metal Liquid*
}

\author{
By K. TSUMURAYA, ** M. S. WATANABE ${ }^{* *}$ and Sinai K. IKEDA**
}

\begin{abstract}
Synopsis
Molecular dynamics is used to study the homogeneous nucleation and growth processes for the sodium system of 864 atoms with constant volume. The equilibrium liquid at $381 \mathrm{~K}$ has been cooled directly to $181 \mathrm{~K}$. The processes have been monitored by studying mean-square displacement of atoms, potential energy, and pair-distribution functions. The supercooled liquid is found to crystallize into bcc structure. The number of atoms in the critical nucleus is at least greater than 43 atoms which is almost coincident with prediction from the classical nucleation theory. We have obtained the solid-liquid interfacial energies from the local pressures of each atom. The energies increase with increasing cluster size. The nucleus is found to advance at a rate of $187 \mathrm{~m} \mathrm{~s}^{-1}$, which is extremely larger than the rate from the kinetic theory for growth.
\end{abstract}

Key words: simulation; molecular dynamics; homogeneous nucleation; growth rate sodium; size dependent interfacial energy.

\section{Introduction}

Molecular dynamics simulation (MD) has been widely used to study microscopic features of materials. One of the application of the MD method has been to simulate the glass formation process of liquid by quenching. Since no pure metal has succeeded in glass formation by quenching in laboratories, this has been a useful method to study the glass structure of single component systems. On the other hand, in 1976 Mandell, McTague and Rahman ${ }^{1)}$ have first reported observation of homogeneous nucleation of crystal in MD method of a supercooled Lennard-Jones liquid. Since then, considerable work has been published concerning observations of homogeneous nucleation in which a variety of interaction potential has been used: the Lennard-Jones (LJ) 12 6 potential, ${ }^{1-3)}$ the soft core potential, ${ }^{4,5)}$ rubidium system, ${ }^{6,7)}$ and sodium system. ${ }^{8)}$ In those studies, one of the motivation was to gather evidence regarding the nucleation phenomenon.

This paper reports the results of a molecular dynamics study of homogeneous nucleation and growth in a model system of liquid sodium, in which we analyze the solid-liquid interfacial energy and arrangement of atoms during the nucleation. In Chap. $I I$ we will describe computational procedure and cooling method. In Chap. III nucleation and growth processes will be given. In Chap. IV we will analyze the particle size dependence of solid-liquid interfacial energies in detail. Discussion and concluding remarks will be given in Chaps. $V$ and $V I$.

\section{Computational Outline}

The pair-interaction potential used in our calculation is the long-range oscillatory potential for sodium. ${ }^{9}$ ) The potential is rubidium-like and typical for simple metals. The pair-distribution functions (PDF) which have been obtained by the potential have coincided with experimental PDF. ${ }^{10}$ The potential has been used recently in our previous papers to study the glass formation process $^{8)}$ and the glass transition ${ }^{11)}$ of the system. The system contains 864 atoms with the three-dimensional periodic boundary condition in order to avoid the surface effect. The density of the system is held at $0.920 \times 10^{3} \mathrm{kgm}^{-3}$ through the calculations. The movement of atoms has been simulated in dimensionless form by use of the following units: the unit of length $\sigma=0.324 \mathrm{~nm}$ where the potential crosses the abscissa for the first time, the unit of mass $m=3.82 \times$ $10^{-26} \mathrm{~kg}$, and the unit of energy $\varepsilon=599 k_{\mathrm{B}}$ where $k_{\mathrm{B}}$ is Boltzmann's constant. The unit of pressure $p_{u}=\varepsilon \sigma^{-3}$ and the unit of the interfacial energy $\gamma_{u}=\varepsilon \sigma^{-2}$. The unit of time $\tau_{u}=6.96 \times 10^{-13} \mathrm{~s}$ which is given by $\left(m \sigma^{2} \varepsilon^{-1}\right)^{1 / 2}$. The time step $\Delta t$ of integration is $5.00 \times$ $10^{-15} \mathrm{~s}$. We have constructed the perfect fcc structure with the density and then displaced the atom positions into random directions. Initial temperature has been chosen to be $381 \mathrm{~K}$, which is higher than the melting temperature $T_{m}$ by $10 \mathrm{~K}$. In order to hold the system at the temperature, the velocities of the atoms have been controlled by use of a modified momentum scaling method. ${ }^{12)}$ We have simulated the time development of atom positions at the temperature for 4000 steps in order to obtain an equilibrium liquid. The velocities have been integrated by use of the Beeman method. ${ }^{13)}$ The system has been cooled directly to $181 \mathrm{~K}$ by use of the scaling method which has controlled temperature of the system in several steps. The system has been simulated for 20000 steps by use of the method in order to observe the nucleation and growth processes at the temperature. The temperature fluctuation has been $180.7 \pm 0.7 \mathrm{~K}$. The positions and velocities of the atoms have been recorded for every five-time step.

\section{Nucleation and Growth Processes}

First, let us clarify when the crystallization has begun in the supercooled liquid. The mean-square dis-

* Based on the paper presented to the 1987 Autumn Meeting of The Japan Institute of Metals, October 1987, at Kumamoto Institute of Technology in Kumamoto. Manuscript received on February 15, 1988; accepted in the final form on April 15, 1988. (C) 1988 ISIJ

** School of Engineering, Meiji University, Higashi-Mita, Tama-ku, Kawasaki 214. 
placement has been a measure of migration of atoms in the liquid state. The displacement is given by

$$
R^{2}(t)=\frac{1}{\mathcal{N}} \sum_{i=1}^{N}\left\{r_{i}(t)-r_{i}(0)\right\}^{2}
$$

where, $r_{i}(t)$ : the position of the $i$-th atom at time $t$

$\mathcal{N}$ : the total number of atoms of the system.

The displacement at $181 \mathrm{~K}$ has been examined. Figure 1 shows the result. The displacement increases almost linearly with time, after having a sharp increment in the initial part. The displacement begins to saturate at about 14000 step and has almost constant values with fluctuation. This implies that the system has crystallized in this stage. The variation of the displacement however seems to give no information about when the system begin to crystallize.

We have obtained the potential energy per atom in the system. Figure 2 shows the variation of the energies as function of time step. The energies, which are fluctuating, are almost constant up to 12500 step. Then the energies begin to decrease drastically between 12500 and 14000 steps. The en-

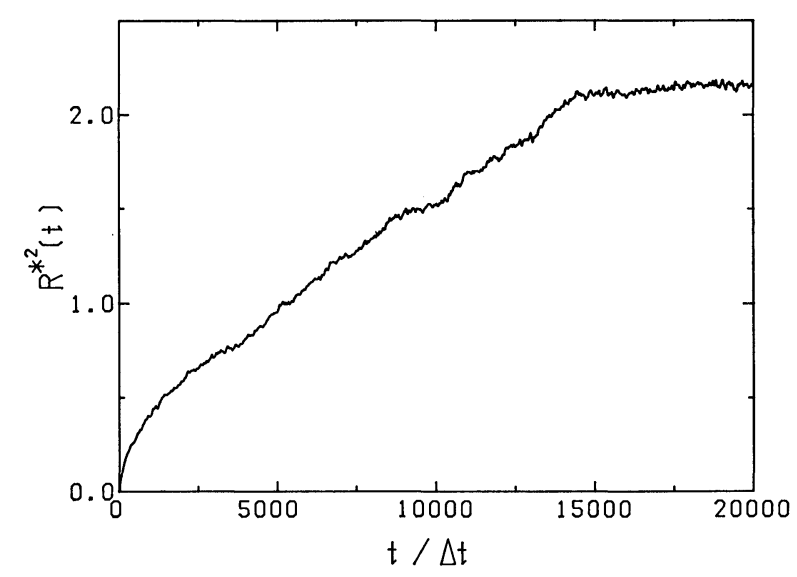

Fig. 1. The mean-square displacement $R^{2}(t)$ per atom in configuration space.

The temperature is $181 \mathrm{~K}$.

It is not the quantity usually referred to as $\left\langle r^{2}\right\rangle$.

The time $\Delta t$ is the molecular dynamics integration step.

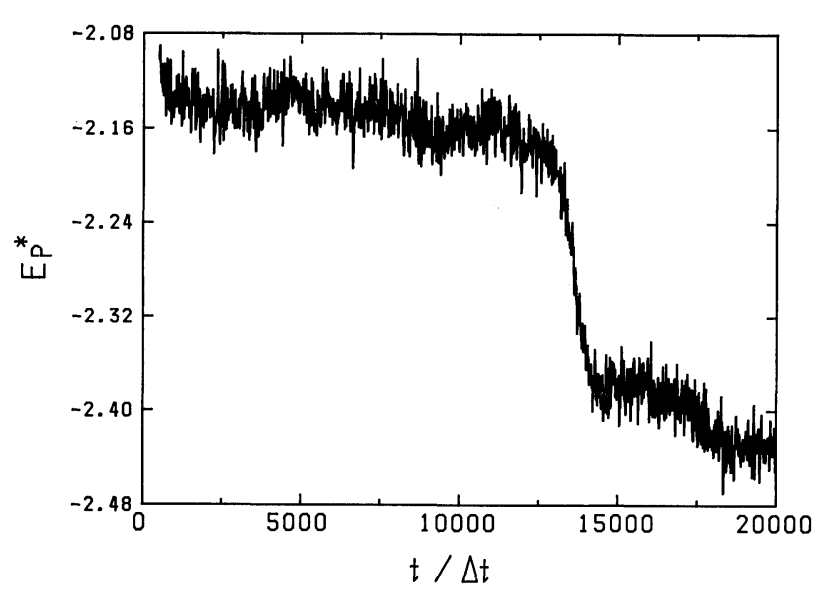

Fig. 2. Variation of potential energy $E_{p}$ during isothermal holding at $181 \mathrm{~K}$. ergies have almost constant values between 14000 and 17500 steps and then decrease slightly. The time step 14000 coincides with the time step that the displacements begin to saturate. Thus the drastic energy decrease implies the initiation of crystallization in the system.

In order to verify the crystallization in the supercooled liquid, the PDF's have been obtained. The PDF's have been calculated by averaging for every 5 step between 12005 and 12100 time steps. This stage is before the drastic energy decrease. Figure 3 (a) shows the result. The PDF is liquid-like; the second peak is broad and has no sub-peak. ${ }^{11)}$ Another PDF has been calculated in the same way between 15005 and 15100 steps which is just after the energy decrease. The PDF is shown in Fig. 3(b). The second peak has a sub-peak on the inner side of the peak, while the shape of the first peak is unchanged. The previous paper has explained the characteristic feature of PDF with bcc structure ${ }^{8)}$ : the sub-peak of the second peak is attributed to the third nearest-neighbor atoms and the main second peak corresponds to the fourth and fifth nearest-neighbor atoms in the structure. The change
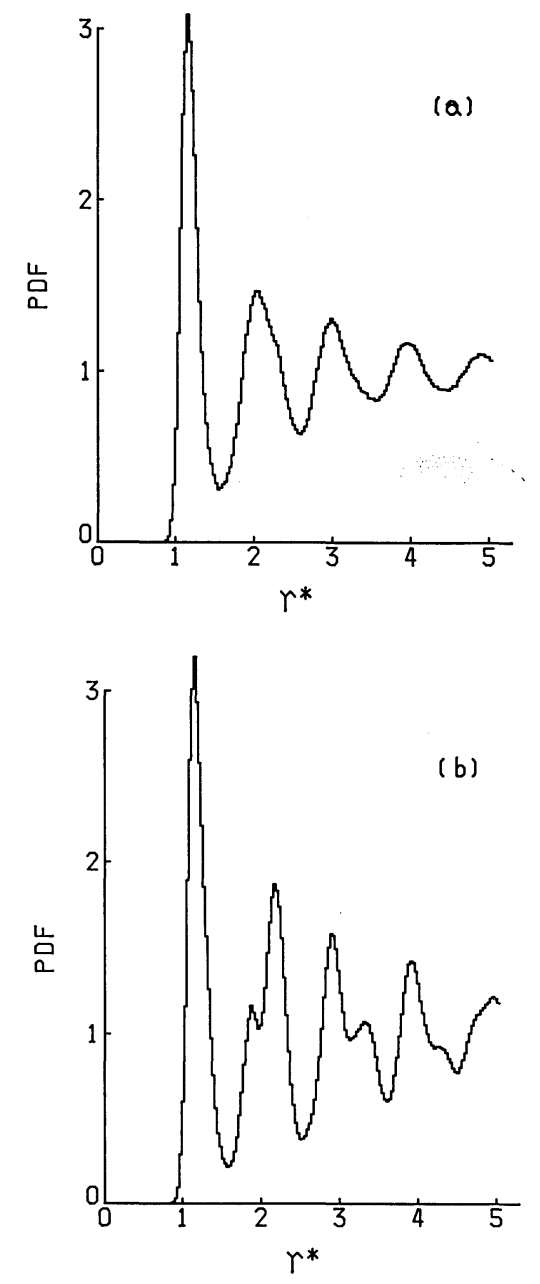

(a) Before potential energy decrease between 12005 and 12100 steps

(b) After potential energy decrease between 15005 and 15100 steps

Fig. 3. Pair-distribution function obtained from every 5 step. 
of the PDF's from Figs. 3(a) to 3(b) indicates that the system has crystallized into bcc structure during the potential energy decrease.

The crystallization process has been analyzed in more detail. Figure 4 shows the trajectories of atoms which have projected the positions of atoms into the $X-Y$ plane of the cubic simulation cell. Figures $4(\mathrm{a})$ and $4(\mathrm{~b})$ are just before the drastic energy decrease, (c) and (d) are in the midst of the decrease and the rest are after the decrease. The PDF corresponded to Fig. 4(a) has been shown in Fig. 3(a). In Figs. 4(a) and $4(\mathrm{~b})$ the trajectories of atoms are almost at random, although a part of the atoms seem to coagulate. The coagulation increases in Fig. 4(c). In Fig. 4(d) a part of the atoms has an ordered structure. The structure expands from (e) to (h). Some atoms still have high mobility in the last figure (h), indicating the structure has lattice defects such as vacancies and interstitials.

It is possible to estimate roughly the rate for growth of the crystal. The length of the molecular dynamics box is $10.18 \sigma$. The growth occurred in $1500 \mathrm{dt}$. Assuming that the critical nucleus to be zero at the initiation of growth, we obtain the rate to be $220 \mathrm{~m} \mathrm{~s}^{-1}$. This may be an overestimated values, since the calculation assumed the initial radius of nucleus to be zero. Walker ${ }^{14)}$ has measured the rates for cobalt and nickel. Their rats have increased with increasing degree $\Delta T$ of supercooling: in the case of cobalt, the rate was $120 \mathrm{~m} \mathrm{~s}^{-1}$ at $\Delta T / T_{m}=0.14$.
Let us see how the crystallization proceeds in an atomistic scale. First of all we must identify the solidlike atoms in the course of crystallization process. Voronoi polyhedron analysis has been used for this purpose. ${ }^{4,8)}$ This analysis however requires a large amount of computing time. Honeycutt and Andersen have reported another method which has been used to identify the solid-like fcc atoms in a supercooled liquid. ${ }^{15}$ ) They defined the clusters in the liquid as follows. In the perfect fcc lattice each atom has 12 nearest-neighbors which contain 6 distinct pairs of neighbors. They form a linear triplet with a central atom. The criterion for deciding a given atom to be solid-like was that the atom must have at least 5 distinct pairs of its 12 nearest-neighbors with which it forms a triplet at an angle greater than a specified angle near $180^{\circ}$. They proposed the optimum angle to be $160 \sim 165^{\circ}$. Here we can apply their method to identify the solidlike atoms in the present system.

In the case of perfect bcc lattice each atom has 8 nearest-neighbors and has 6 second nearest-neighbors. The second nearest-neighbor distance is only 1.15 times as large as the first one, indicating that each atom has 14 neighbors in a narrow range. Seven distinct pairs of neighbors form their linear triplet with the central atom. We have identified the atoms to be solid-like when the atoms form 7 triplets whose angle is greater than $164^{\circ}$ and whose pair distance is less than $0.5 \mathrm{~nm}$. The distance is between the second and the third nearest neighbor distances. We have identified the

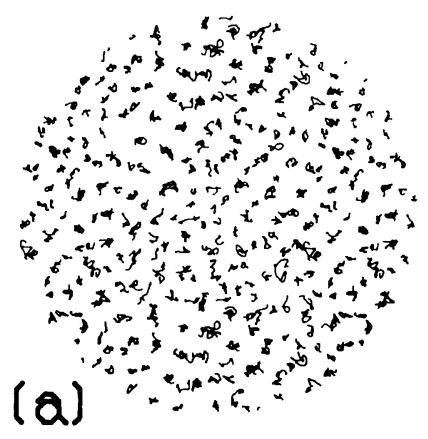

$12005-12250$

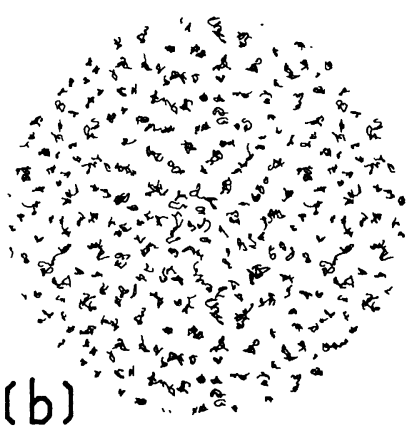

$12255-12500$

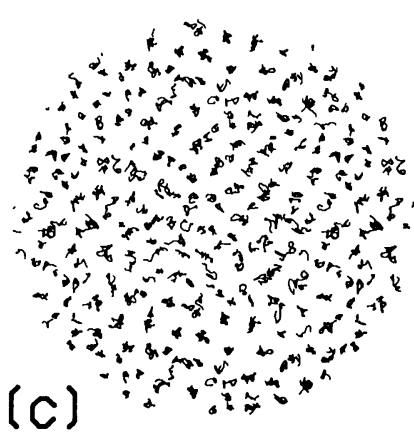

$12505-12750$

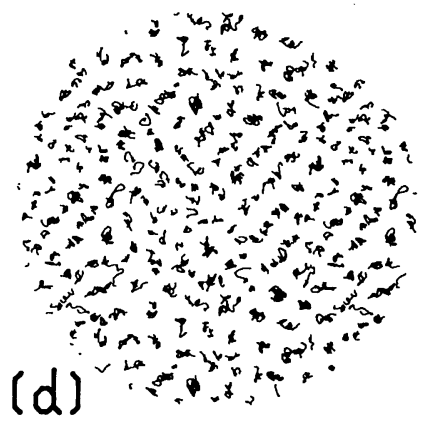

$12755-13000$

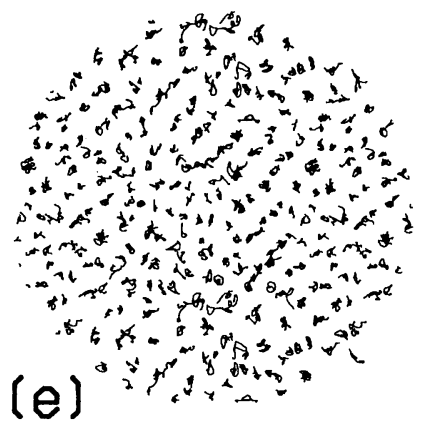

$13005-13250$

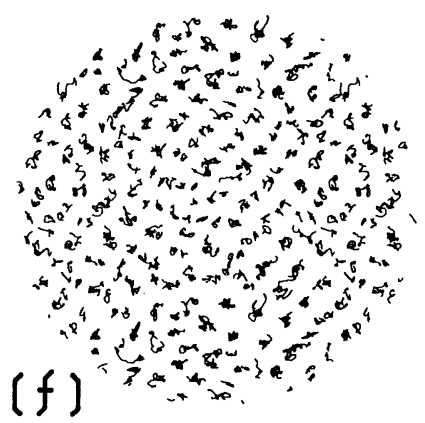

13255-13500

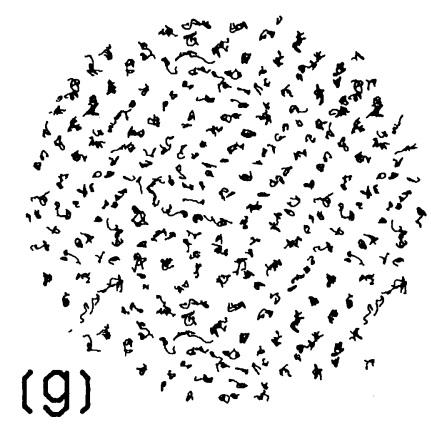

13505-13750

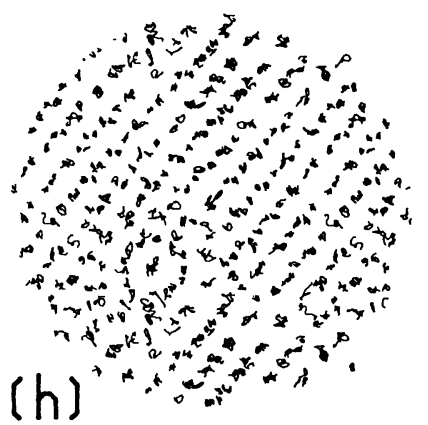

13755-14000

Fig. 4. Trajectories of atoms just before and after the drastic decrease of potential energy of Fig. 2 where the positions are projected to the $X-Y$ plane of the cubic simulation cell. 
atoms without overlapping. The obtained number of the atoms has been found almost in coincidence with that with Voronoi analysis. Figure 5 shows a series of perspective figures of the atoms. The shot indicated by 12100 has been obtained as follows: the positions of each atom have been averaged over between 12055 and 12150 steps. After then we have identified the solid-like atoms. The number 12100 is thus the central step of the averaged 100 steps. A series of the shots is shown for every 100 step. There are several solid-like clusters in figure 12 100: the atom group on the left side wall belongs to the larger cluster on the right bottom, since the simulation cell has the periodic boundary condition in three dimensions. Figure 12100 thus contains 3 clusters. In figure 12200 two of the clusters however have annihilated and there is only one cluster. In 12300 the cluster has moved to the upper right corner. In 12400 there are 4 clusters. Such types of construction and annihilation continue up to figure 12700 . The clusters in figure 12700 do not annihilate in the next figure 12800 . The size of the cluster has remained almost constant up to 12900 , although it is fluctuating. The cluster however begins to grow from figure 13100

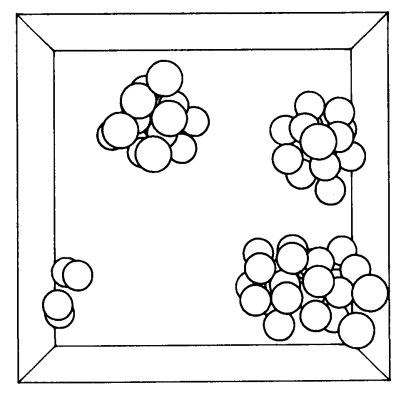

12100

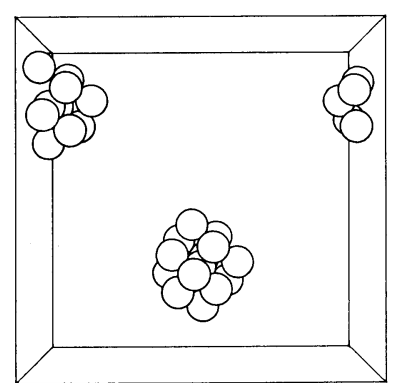

12500

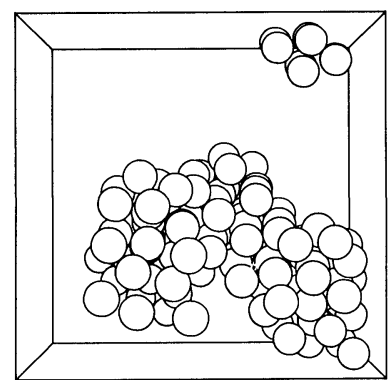

12900

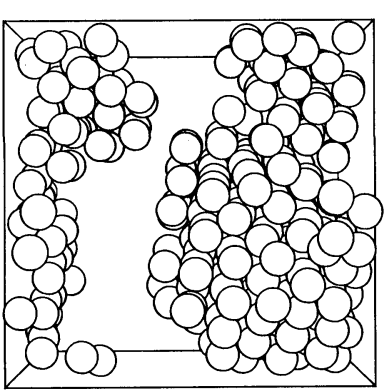

13300

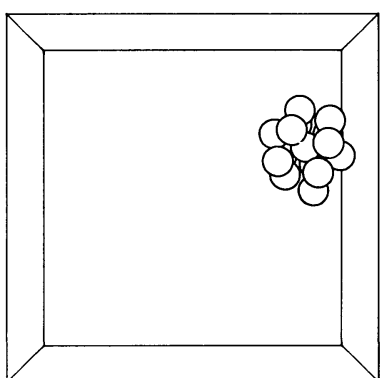

12200

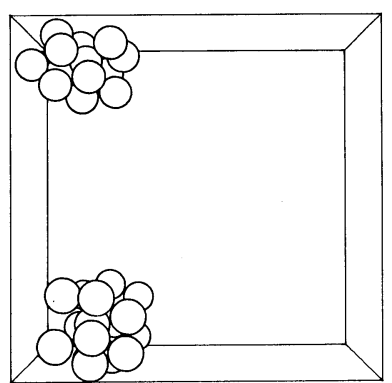

12600

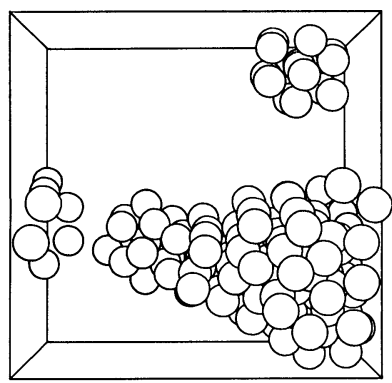

13000

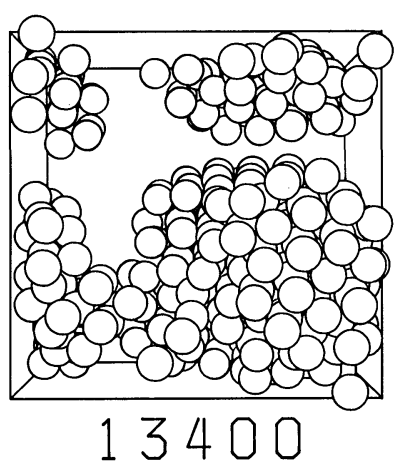

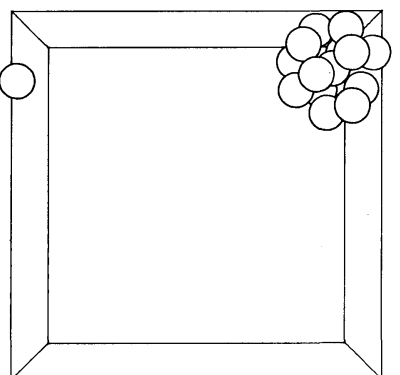

12300

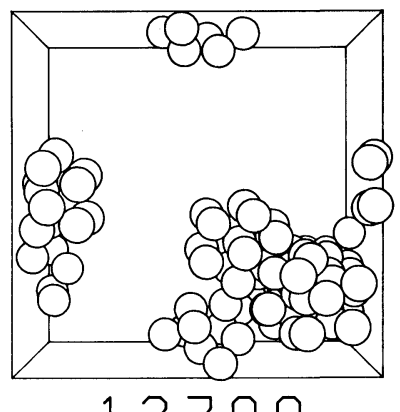

12700

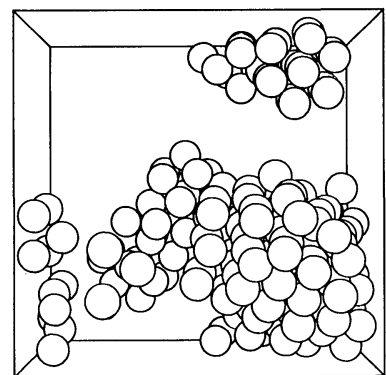

13100

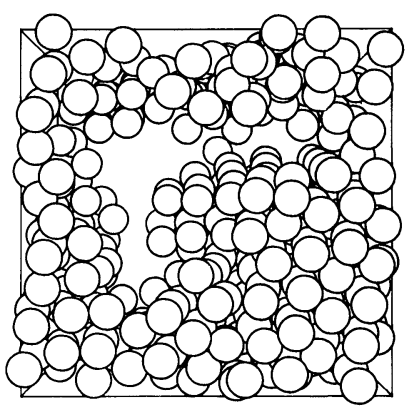

13500
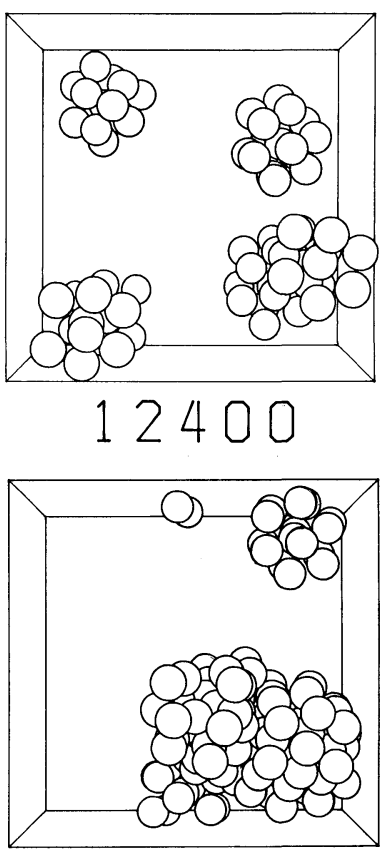

12800

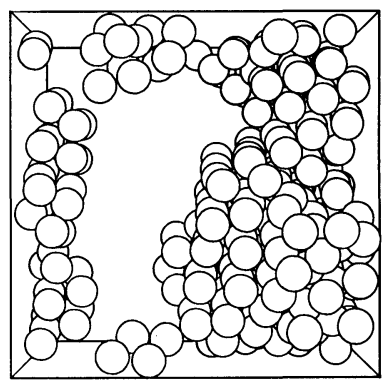

13200

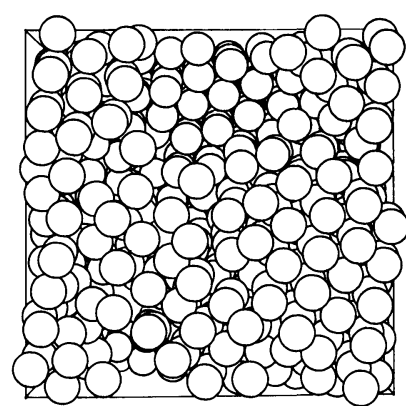

13700

Fig. 5. The perspective figures of the solid-like atoms. The positions of the atoms have been obtained from averaging over 100 steps. 
which means that the cluster has exceed a critical size $r^{*}$. The nucleus of crystal continue to grow into the liquid region. In the figure 13700 all the atoms have crystallized.

Here we can estimate roughly the critical number of atoms for nucleation. In order to know the variation of the number of atoms in the cluster, the numbers have been plotted as a function of time steps. Figure 6 shows the result. The numbers are fluctuating between 40 and 50 atoms up to 12500 step. It begins to grow drastically after then. There seems to exist a critical size for nucleation at around 12500 step. Assuming that the crystal grows spherically with a radial rate, we obtain the following equation.

$$
\mathcal{N}^{1 / 3}=\mathcal{N}_{0}^{1 / 3}+(4 \pi \rho / 3)^{1 / 3}\left(t-t_{0}\right) v,
$$

where, $\mathcal{N}$ : the number of atoms at time $t$

$\mathcal{N}_{0}$ : the number of atoms at time $t=t_{0}$ which corresponds to the critical number of atoms

$\rho:$ the number density of atoms

$v$ : the velocity of the solid-liquid interface. We have plotted $\mathcal{N}^{1 / 3}$ as a function of time step in order to obtain $\mathcal{N}_{0}$ and $v$. Figure 7 shows the result.

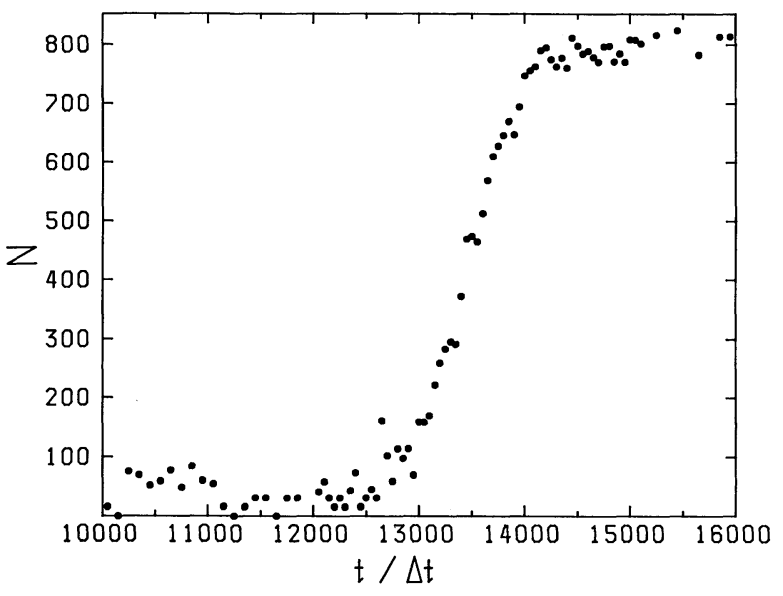

Fig. 6. The number $\mathcal{N}$ of atoms contained in the nucleus as a function of time.

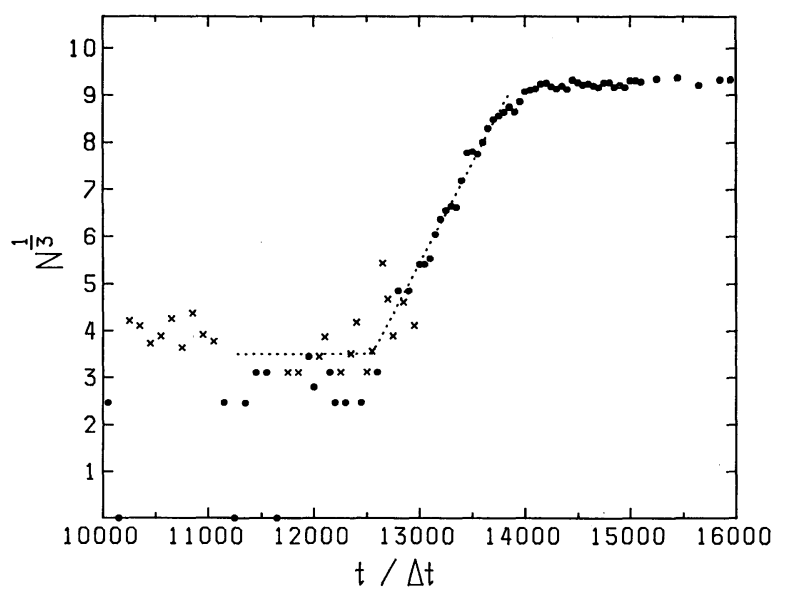

$x:$ The numbers of atoms when there have been more than two nuclei in the system .....: Visual guidance

Fig. 7. Variation of $\mathcal{N}^{1 / 3}$ as a function of time.
In order to estimate the number of atoms in the critical radius, we have plotted the quantities $\mathcal{N}^{1 / 3}$ as open circles when there is a single cluster in the snap shots. A linear relationship has been obtained for growth process. The slope gives the growth velocity to be $187 \mathrm{~m} \mathrm{~s}^{-1}$. The cluster has not grown even when $\mathcal{N}_{0}^{10 / 3}$ $=3.5$ at about 12000 step. This indicates the number $\mathcal{N}_{0}$ in the critical radius is at least greater than this value.

\section{Solid-Liquid Interfacial Energy}

The presence of the solid-liquid interfacial energy makes embryo in supercooled liquid unstable. Here we will clarify how the energy changes with growth of the nucleus. In the case of two phase system of solid and liquid, the pressure difference $p_{s}-p_{l}$ between the solid and liquid phases can be given by the Laplace equation as $^{16)}$

$$
p_{s}-p_{l}=\frac{2 \gamma}{r}
$$

where, $\gamma$ : the solid-liquid interfacial energy.

In order to obtain the energy we must know the pressures $p_{s}$ and $p_{l}$.

Born and Huang ${ }^{17)}$ have developed an equation to evaluate the local stress $\sigma_{i}^{p q}$ for $i$-atom as

$$
\sigma_{i}^{p q}=\frac{1}{2 \Omega_{i}} \sum_{j} \frac{\partial}{\partial r_{i j}} \phi\left(r_{i j}\right) \frac{r_{i j}^{p} r_{i j}^{q}}{\left|r_{i j}\right|}
$$

where, $\sigma_{i}^{p q}$ : the local atomic level stresses at the position of atom $i$

$p q$ : the component of the stress

$r_{i j}$ : the vector $r_{i}-r_{j}$ in which $r_{i}$ and $r_{j}$ are the position vectors of atoms $i$ and $j$

$r_{i j}^{p}:$ the $p$ component of the vector $r_{i j}$

$\Omega_{i}$ : the atomic volume of the $i$-th atom

$\phi:$ the two-body potential.

The choice of the coordinate system is arbitrary since the stress is explicitly dependent on this choice. In the present calculation of $\sigma_{i}^{p q}$ the volume $\Omega_{i}$ has been calculated by the procedure ${ }^{8)}$ of the Voronoi polyhedron analysis. From the three principle stresses $\sigma_{1}, \sigma_{2}$, and $\sigma_{3}$ we can obtain the pressure $p$ as

$$
p=\frac{1}{3}\left(\sigma_{1}+\sigma_{2}+\sigma_{3}\right)
$$

Thus the pressure $p$ is the locally hydrostatic pressure which can be obtained for each particle.

The pressure $p$ for each atom has been obtained from time-averaged positions over 100 steps. The total pressure $p^{*}$ has about $1.7 p_{u}$ during embryo formation process and has increased to $2.4 p_{u}$ after crystallization. The pressure $p_{s}$ of the nuclei has been obtained by averaging over the atoms which belong to the solid-like cluster. The atoms in the cluster have been already identified in Fig. 6. The pressure $p_{l}$ in the liquid has been obtained by the same way. Thus the solid-liquid interfacial energy $\gamma$ has been obtained. Figure 8 shows the result. Although the energies are fluctuating, the energies seem to increase with time. 


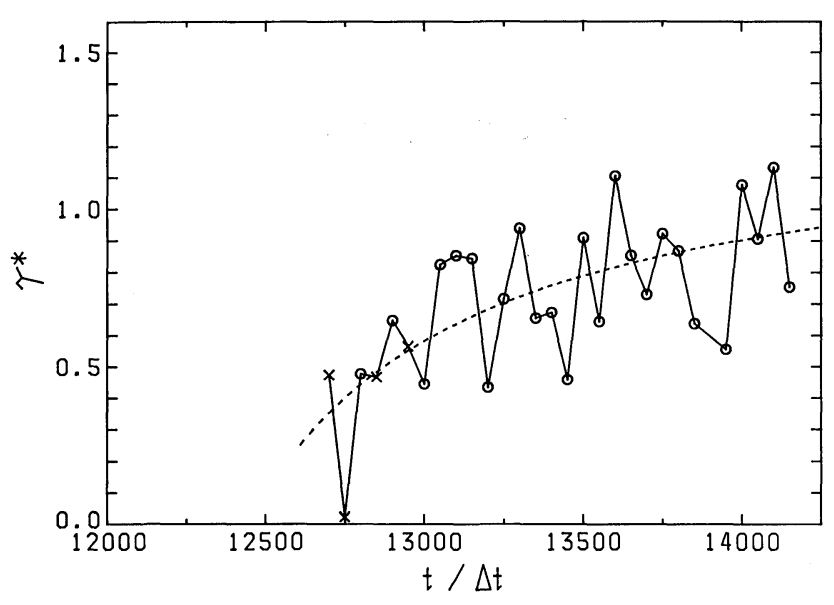

-.-: The size dependence of Eq. (6) with $\delta=$ $0.32 \mathrm{~nm}$ and $\gamma_{\infty}=1.3$ (for sodium this value corresponds to $102 \mathrm{~mJ} \mathrm{~m}^{-2}$ ).

Fig. 8. Variation of the solid-liquid interfacial energies as a function of time.

Ono and Kondo proposed an expression for a curvature-dependent solid-liquid interfacial energy ${ }^{18)}$ as

$$
\gamma=\gamma_{\infty}\left[1-\frac{2 \delta}{r}\right]
$$

where $\delta$ the distance between the Gibbs surface of tension and the equimolecular surface and is a rough measure of the thickness of the interface.

A broken line in Fig. 8 is drawn for $\delta=0.32 \mathrm{~nm}$ and $\gamma_{\infty}=1.3$ in dimensionless value, or $\gamma_{\infty}=102 \mathrm{~mJ} \mathrm{~m}^{-2}$. The magnitude for $\delta$ is almost equal to the atomic radius for sodium. It has been found in Fig. 7 that the number of atoms for the critical nucleus is at least greater than 43 atoms at which the interfacial energy is $13 \mathrm{~mJ} \mathrm{~m}^{-2}$. Thus the interfacial energy for the critical radius is at least greater than this value. Experimental solid-liquid interfacial energy has been obtained as $20 \mathrm{~mJ} \mathrm{~m}^{-2}$ by an undercooling experiment. ${ }^{19)}$

\section{Discussion}

It is instructive to clarify whether the classical nucleation theory is compatible with the computer observations. The excess energy $\Delta G$ of a nucleus is given by

with

$$
\Delta G=n \Delta \mu+4 \pi r\left(\frac{3 n}{4 \pi \rho}\right)^{2 / 3},
$$

Equation (7) has a positive maximum $W^{*}$ for $n=n^{*}$ which is the critical number of atoms in the critical radius. Thus the number $n^{*}$ can be estimated as 38 atoms when we use $\gamma=20 \mathrm{~mJ} \mathrm{~m}^{-2} \cdot{ }^{19)}$ This is almost equal to the minimum number of atoms in the critical nucleus given in Fig. 7.

According to a kinetic model for crystal growth, the maximum growth velocity $V_{\max }$ is given by ${ }^{20)}$

$$
V_{\max }=m_{i} \frac{\Delta \mu}{d_{i}},
$$

where, $m_{i}$ : the mobility of the component in liquid $\Delta \mu:$ the chemical potential difference between liquid and solid states

$d_{i}$ : the distance of the solid-liquid interface. Taking $d_{i}=\delta$ in Eq. (6) we obtain $V_{\max }=0.304 \mathrm{~m} \mathrm{~s}^{-1}$ at $181 \mathrm{~K}$. This is extremely smaller than the velocity $187 \mathrm{~m} \mathrm{~s}^{-1}$ obtained from the present simulation. This implies that the kinetic theory should be modified so as to predict this magnitude of the velocity.

\section{Concluding Remarks}

Nucleation and growth processes have been studied for the sodium system with 864 atoms by molecular dynamics simulation with a constant volume. The equilibrium liquid has been formed at $381 \mathrm{~K}$ and cooled to $181 \mathrm{~K}$ by the momentum scaling method. The crystallization has been confirmed by the variation of potential energy. The crystal structure has been identified to be bcc through the shape change of the pair-distribution functions. We have observed the construction and destruction process of cluster embryos and the growth process in supercoold liquid. The number of atoms in the critical nucleus is at least greater than 43 atoms. This value is almost coincident with prediction from the classical nucleation theory. We have obtained the solid-liquid interfacial energies from the pressure difference between liquid and solid regions using the local pressures for each atom. The energies have increased with increasing cluster size. The energy is $13 \mathrm{~mJ} \mathrm{~m}^{-2}$ for the 43 atom cluster which is comparable in magnitude to the experimental value $20 \mathrm{~mJ} \mathrm{~m}^{-2}$. The growth rate is found to be $187 \mathrm{~m} \mathrm{~s}^{-1}$, while the kinetic theory predicts the rate to be only $0.3 \mathrm{~m} \mathrm{~s}^{-1}$.

\section{Acknowledgements}

The authors thank the Computer Center, Meiji University for the use of the FACOM M-380S computer and the Computer Center, Institute for Molecular Science, Okazaki National Research Institute for the use of the HITAC M-680H and S810/10 computers.

\section{REFERENCES}

1) M. J. Mandell, J. P. McTague and A. Rahman: J. Chem. Phys., 64 (1976), 3699.

2) M. J. Mandell, J. P. McTague and A. Rahman: J. Chem. Phys., 66 (1977), 3070.

3) G. S. Hsu and A. Rahman: J. Chem. Phys., 71 (1979), 4974.

4) M. Tanemura, Y. Hiwatari, H. Matsuda, T. Ogawa, N. Ogita and A. Ueda: Prog. Theor. Phys., 58 (1977), 1079.

5) J. N. Cape, J. L. Finny and L. V. Woodcock: J. Chem. Phys., 75 (1981), 2366.

6) G. S. Hsu and A. Rahman: J. Chem. Phys., 70 (1979), 5234.

7) R. D. Mountain and P. K. Basu: J. Chem. Phys., 78 (1983), 7318.

8) M. S. Watanabe and K. Tsumuraya: J. Chem. Phys., 87 (1987), 4891.

9) M. D. Jhonson, P. Huchonson and N. H. March: Proc. Roy. Soc. London, Ser. A, 282 (1964), 283. 
10) M. Tanaka, Y. Fukui and S. Takeuchi: J. Jpn. Inst. Met., 37 (1973), 907.

11) M. S. Watanabe and K. Tsumuraya: J. Chem. Phys., 88 (1988), 1991.

12) M. Tanaka: J. Phys. Soc. Jpn., 51 (1982), 3802.

13) P. Schfield: Comp. Phys. Commun, 5 (1973), 17. D. Beeman: J. Comp. Phys., 20 (1976), 130.

14) J. L. Walker: results are published in B. Chalmers, Principles of Solidification, J. Wiley \& Sons, New York, (1954), 114-115.

15) J. D. Honeycutt and H. C. Andersen: J. Phys. Chem., 90
(1986), 1585.

16) L. E. Murr: Interfacial Phenomena in Metal and Alloys, Addison-Wesley Pub. Co., London, (1975), 87.

17) M. Born and K. Huang: Dynamical Theory of Crystal Lattices, Clarendon Press, Oxford, (1954).

18) S. Ono and S. Kondo: Handbuch der Physik, X, ed. by S. Flugge, Springer-Verlag, Berlin, (1960) 153.

19) D. Turnbull: J. Chem. Phys., 18 (1950), 769.

20) K. A. Jackson: Liquid Metals and Solidification, Am. Soc. Met., Gleveland, (1958), 174. 